\title{
Síndrome da apneia obstrutiva do sono como risco independente de doenças cerebrovasculares
}

\author{
Christianne M.C.S. Bahia, ${ }^{1,2}$ João S. Pereira, ${ }^{1,3}$ Andréia Brandão ${ }^{3,4}$
}

\begin{abstract}
Resumo
A síndrome da apneia obstrutiva do sono (SAOS) tem sido extensivamente pesquisada nas últimas duas décadas, permitindo uma maior compreensão sobre sua relevância na prática clínica. Trata-se de uma doença com alta prevalência, acometendo até um terço da população adulta. A SAOS tem sido associada a condições sabidamente envolvidas no aumento do risco de eventos cerebrovasculares, como a hipertensão arterial sistêmica, diabetes mellitus tipo 2, obesidade e fibrilação atrial. Estudos recentes demonstram que a SAOS, por si só, pode levar à lesão cerebral isquêmica, possivelmente por alterações hemodinâmicas no fluxo cerebral, assim como alterações inflamatórias que promovem um estado de hipercoagulabilidade e aterosclerose na região encefálica. Ainda não estão totalmente esclarecidos os exatos mecanismos, mas há cada vez mais evidências de que a SAOS pode ser um fator de risco independente para o desenvolvimento da doença cerebrovascular.
\end{abstract}

Descritores: Sono; Síndromes da apneia do sono; Transtornos cerebrovasculares; Fatores de risco.

\begin{abstract}
Obstructive sleep apnea syndrome as an independent risk factor for cerebrovascular disease

Obstructive sleep apnea syndrome (OSA) has been extensively researched in the past two decades, allowing a greater understanding of its relevance in clinical practice. It's a high prevalent disease and can affects around one third of adult population. It has been associated with conditions known to be involved in the increased risk of cerebrovascular events, such as hypertension, diabetes mellitus type II, obesity and atrial fibrillation. Recent studies show that OSA, by itself, can lead to ischemic brain damage, possibly due to hemodynamic changes in cerebral blood flow as well as inflammatory changes that promote a hypercoagulable state and atherosclerosis in the cerebral region. The exact mechanisms are still not entirely clear, but there is increasing evidence that OSA may be an independent risk factor for the development of cerebrovascular disease.
\end{abstract}

Keywords: Sleep; Sleep apnea syndromes; Cerebrovascular disorders; Risk factors.
1. Serviço de Neurologia. Hospital Universitário Pedro Ernesto. Universidade do Estado do Rio de Janeiro. Rio de Janeiro, RJ, Brasil.

2. Serviço Interdisciplinar de Medicina do Sono. Hospital Universitário Pedro Ernesto. Universidade do Estado do Rio de Janeiro. Rio de Janeiro, RJ, Brasil.

3. Faculdade de Ciências Médicas. Universidade do Estado do Rio de Janeiro. Rio de Janeiro, RJ, Brasil.

4. Serviço de Cardiologia. Hospital Universitário Pedro Ernesto. Universidade do Estado do Rio de Janeiro. Rio de Janeiro, RJ, Brasil.

*Endereço para correspondência:

Serviço de Neurologia, HUPE

Boulevard 28 de Setembro, 77, 20 andar

Rio de Janeiro, RJ, Brasil. CEP: 20551-030

E-mail: : christiannemartins@gmail.com

Revista HUPE, Rio de Janeiro, 2016;15(1):56-60

doi: 10.12957/rhupe.2016.22375

Recebido em 18/01/2016. Aprovado em 28/03/2016.

\section{Resumen}

Apnea del sueño obstructiva como riesgo independiente para la enfermedad cerebrovascular

La síndrome de apnea obstructiva del sueño (SAOS) ha sido investigado ampliamente en las últimas dos décadas, lo que permite una mayor comprensión de su relevancia en la práctica clínica. SAOS afecta hasta un y ha sido asociado con condiciones conocidas por estar implicado en el aumento del riesgo de eventos cerebrovasculares, tales como hipertensión, diabetes mellitus tipo 2, la obesidad y la fibrilación auricular. Estudios recientes muestran que la SAOS, por sí mismo, puede conducir a daño cerebral isquémico, posiblemente debido a los cambios hemodinámicos en el flujo sanguíneo cerebral, así como los cambios inflamatorios que promueven un estado de hipercoagulabilidad y la aterosclerosis en la región cerebral. Los mecanismos exactos no están del todo claras, pero cada vez hay más pruebas de que la SAOS puede ser un factor de riesgo independiente para el desarrollo de la enfermedad cerebrovascular.

Palabras clave: Sueño; Síndromes de la apnea del sueño; Trastornos cerebrovasculares; Factores de riesgo. 


\section{Artigo de revisão}

\section{Introdução}

A síndrome apneia obstrutiva do sono (SAOS) é caracterizada por episódios recorrentes de obstrução da via aérea superior (VAS) durante o sono, levando à limitação total ou parcial do fluxo de ar, apesar da manutenção do esforço da musculatura respiratória. As principais consequências são hipóxia intermitente, fragmentação do sono, hipercapnia e aumento da ativação simpática, sendo estes os principais mecanismos responsáveis pelas repercussões sistêmicas da SAOS. ${ }^{1}$ (Figura 1)

A prevalência da SAOS é alta, estimada em torno de $22 \%$ (variação de 9 a 37\%) em homens e 17\% (variação de 4 a 50\%) em mulheres. ${ }^{2}$ No Brasil, um estudo recente com 1.042 participantes da cidade de São Paulo entre 20 e 80 anos mostrou que $32,8 \%$ dos participantes apresentavam critérios para SAOS. ${ }^{3}$ A chance de desenvolver SAOS aumenta com a idade, sendo maior em indivíduos acima de 60 anos, obesos e com circunferência cervical aumentada $(\geq 37 \mathrm{~cm}$ em mulheres e $43 \mathrm{~cm}$ em homens). ${ }^{3,4}$

O padrão-ouro para o diagnóstico de SAOS é a polissonografia realizada em laboratório de sono com monitorização neurológica e respiratória de noite inteira. Os critérios para o diagnóstico de SAOS foram recentemente revisados ${ }^{5}$ com o intuito de abranger as novas descobertas sobre esta doença. Os atuais critérios diagnósticos e a classificação da SAOS em adultos encontram-se listados na tabela 1.5,6
Nas últimas duas décadas houve um grande aumento na pesquisa da SAOS, o que contribuiu muito para o avanço no conhecimento sobre esta doença. Muitos estudos reportaram a associação da SAOS com outras condições clínicas envolvidas no aumento do risco de eventos cardiovasculares em geral como a hipertensão arterial, diabetes mellitus tipo 2, fibrilação atrial e obesidade. Porém, quanto mais se estuda a SAOS, mais se desvenda a complexidade de seus efeitos, admitindo-se cada vez mais uma possibilidade de ela própria atuar de forma independente no aumento do risco para doenças cerebrovasculares.

O presente artigo tem como objetivo revisar os principais avanços produzidos nas últimas duas décadas sobre o impacto da SAOS no aumento direto e indireto do risco de eventos cerebrovasculares.

\section{SAOS e hipertensão arterial sistêmica}

A hipertensão arterial é o principal fator de risco modificável para a ocorrência de acidente vascular cerebral isquêmico (AVCi). ${ }^{7}$ A SAOS é considerada um fator de risco independente para o desenvolvimento da hipertensão, havendo uma relação diretamente proporcional entre o índice de apneia e hipopneia (IAH), que reflete a gravidade da doença, e a chance de uma pessoa se tornar hipertensa. ${ }^{8}$

Os prováveis mecanismos fisiopatológicos envolvidos na indução da hipertensão arterial sistêmica em pacientes com SAOS são a hiperatividade simpática, o aumento dos níveis de endotelina-1 (importante subs-

Tabela 1. Classificação e critérios diagnósticos e da SAOS em adultos.

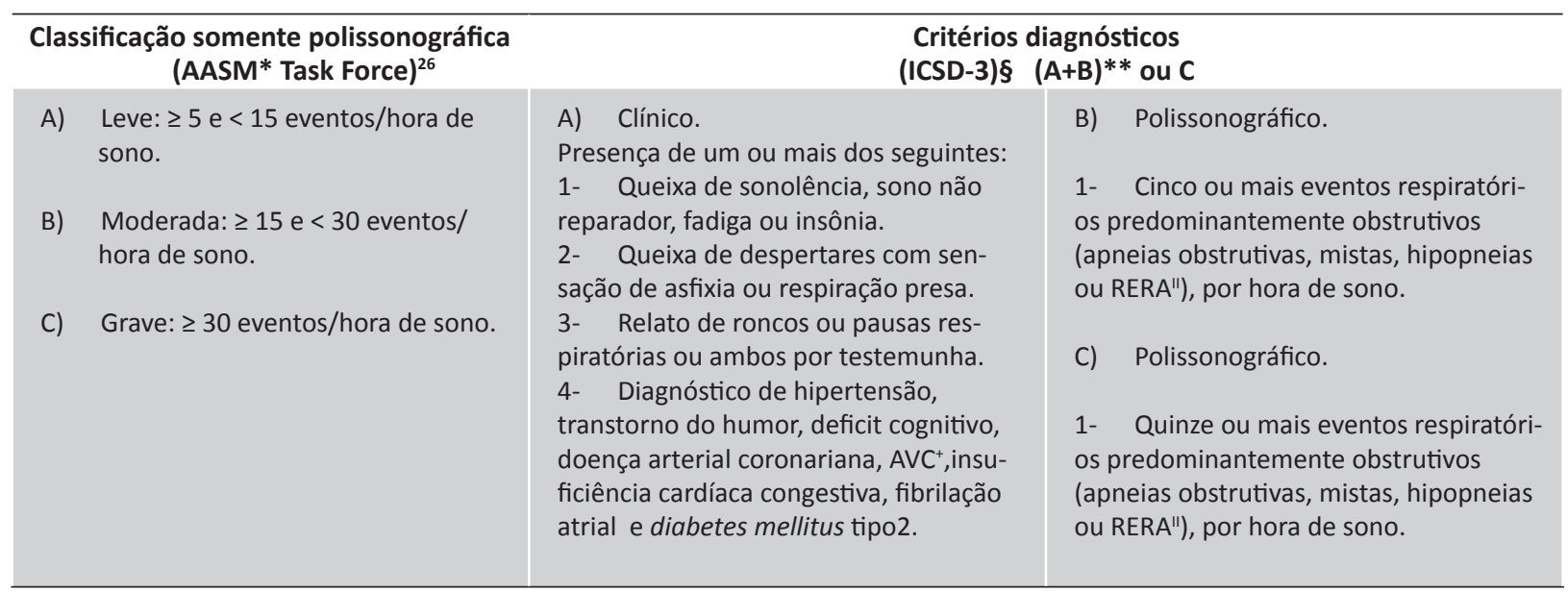

*AASM - American Academy of Sleep Medicine; +AVC - acidente vascular cerebral; II RERA - Respiratory Effort Related Arousals; § ICSD-3: International Classification of Sleep Disorders, Third Edition.

** Pelos critérios ICSD-3, a SAOS leve depende também de sinais e sintomas clínicos (A), além de polissonográficos (B). 
tância vasoconstrictora que é induzida em situações de hipóxia) e a desregulação da secreção de renina e aldosterona, que tem forte influência do ritmo sonovigília, e acaba sendo prejudicada pela fragmentação do sono decorrente dos eventos respiratórios obstrutivos. ${ }^{1}$

\section{SAOS e arritmia cardíaca}

O risco de AVCi é aproximadamente cinco vezes maior em indivíduos portadores de fibrilação atrial (FA) comparado aos não portadores ${ }^{7}$ e há evidências cada vez mais consistentes de que a SAOS propiciaria o surgimento desta e de outras arritmias.

Um grande estudo populacional, The Sleep Health Heart Study (SHHS), mostrou uma maior prevalência de FA, bem como o de taquicardia ventricular não sustentada (TVNS), em pacientes com SAOS grave. ${ }^{9}$ Posteriormente, Monahan e colaboradores, ${ }^{10}$ utilizando dados deste mesmo estudo populacional, fizeram uma análise criteriosa da polissonografia de 57 pacientes que apresentaram FA paroxística e TVNS, observando a existência de evento respiratório nos 90 segundos que precediam o aparecimento da arritmia e demostraram uma chance 17,5 vezes maior ( $95 \%$ IR 5.3-58.4) de encontrar uma das arritmias após um evento respiratório anormal comparado ao mesmo período de respiração normal.
Os prováveis mecanismos envolvidos na arritmogênese em pacientes com SAOS seriam as dessaturações intermitentes, as grandes mudanças na pressão intratorácica e a estimulação dos barorreflexos. ${ }^{10}$

\section{SAOS, obesidade e diabetes}

A obesidade e o diabetes também estão entre os cinco principais fatores de risco para o $\mathrm{AVCi}$, compartilhando o ranking com a hipertensão, o tabagismo e as causas cardíacas.? A SAOS parece facilitar o surgimento da obesidade ao promover alterações na secreção de substâncias envolvidas no controle do apetite e gasto energético, como a leptina e na regulação do metabolismo da glicose e dos lipídeos como a adiponectina. ${ }^{7} \mathrm{~A}$ resistência insulínica também parece ser induzida pela SAOS devido à hiperatividade simpática que estimula a glicogenólise e a gliconeogênese, além de estimular a lipólise, aumentando a quantidade de ácidos graxos livres circulantes. ${ }^{1}$

\section{SAOS como fator de risco independente para doença cerebrovascular}

O AVC afeta em torno de 16,9 milhões de pessoas por ano, levando a óbito 5,9 milhões de doentes. O AVC também é considerado a principal causa de invalidez no

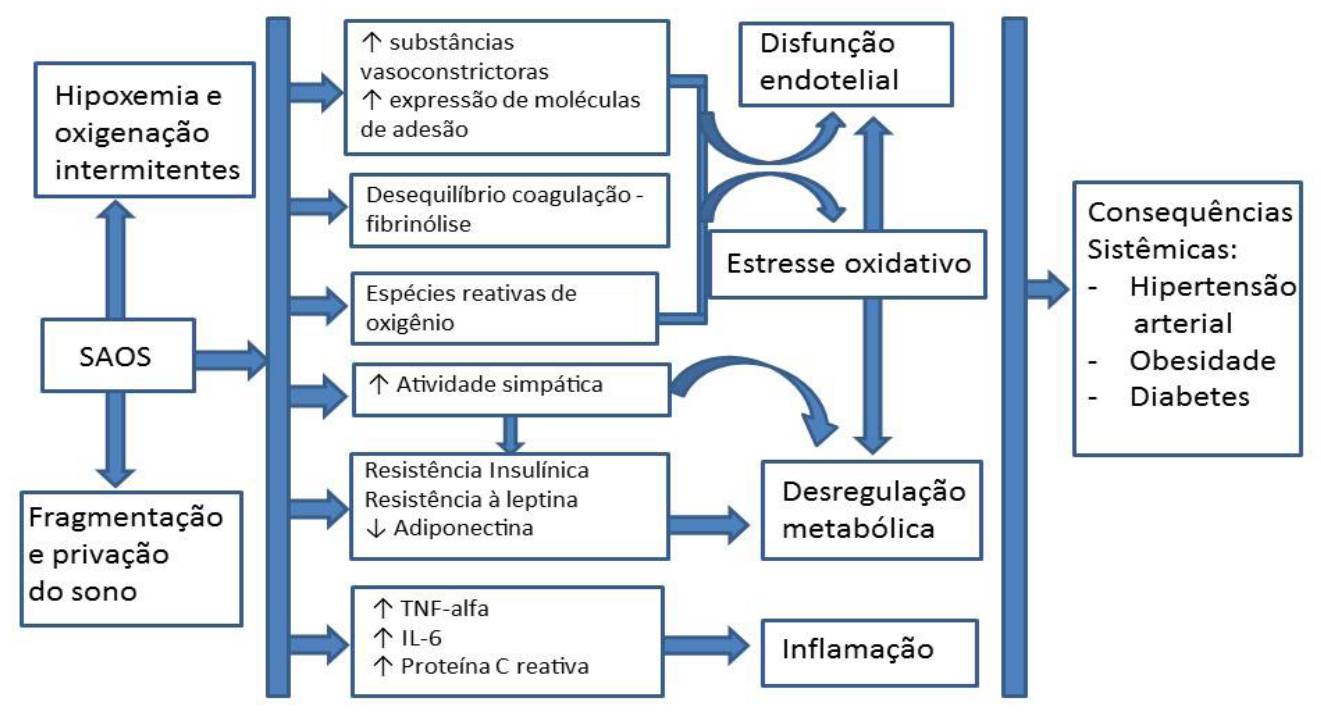

Figura 1. Esquema resumido da cascata de eventos desencadeados pela SAOS. 


\section{Artigo de revisão}

mundo. ${ }^{11}$ Portanto, conhecer os fatores que aumentam a chance de ocorrência de um AVCé essencial para adotar medidas que previnam essa doença tão devastadora.

As evidências de que a SAOS está associada ao maior risco de eventos cerebrovasculares são inegáveis, tanto que a última orientação da American Heart Association (AHA) incluiu como recomendação o rastreio e o tratamento da SAOS em pacientes diagnosticados com AVCi ou ataque isquêmico transitório. ${ }^{12}$

Apesar dos vários mecanismos já expostos pelos quais a SAOS poderia aumentar o risco de AVC indiretamente, vários estudos mostram que ela própria teria um efeito independente neste processo.

Redline e colaboradores ${ }^{13}$ estudaram a incidência de AVC isquêmico em 5.442 pacientes (2.462 homens e 2.960 mulheres) que participaram do SHHS. Esses pacientes foram submetidos à PSG de noite inteira no momento zero e acompanhados por um período médio de 8,7 anos. Neste período, foram observados 193 novos casos de AVCi. A análise dos dados revelou que homens com SAOS moderada a grave (neste estudo defindo como IAH >19,1) apresentavam uma chance 2,86 vezes maior (95\%IC:1,1-7,4 p=0,016), de evoluir com AVCi comparados com homens sem SAOS (IAH<4,05), mesmo após o ajuste das variáveis confundidoras (idade, índice de massa corporal, raça, tabagismo, pressão arterial sistólica, uso de anti-hipertensivos e diabetes). A mesma associação não foi detectada em mulheres neste estudo. Uma possível explicação é que, em geral, mulheres apresentam AVCi em idades mais avançadas que os homens e o acompanhamento de 8,7 anos pode não ter sido suficiente para detectar a ocorrência deste desfecho na população feminina.

Atualmente, um grande estudo multicêntrico com 1.840 pacientes - The Determining Risk of Vascular Events by Apnea Monitoring - DREAM study está sendo conduzido na busca de correlacionar a presença de SAOS com a ocorrência de eventos cardiovasculares, como o AVC e o ataque isquêmico transitório. Os resultados deste estudo podem prover evidências adicionais para o entendimento da associação entre essas duas condições. ${ }^{14}$

Outro objeto de análise diz respeito às alterações na substância branca cerebral, também conhecida como microangiopatia. Estas alterações referem-se a lesões na substância branca subcortical e periventricular, que aparecem com hiperintensidade nas sequências pesadas em T2 e Fluid-atennuated Inversion Recovery (FLAIR) da ressonância nuclear magnética (RNM). Seu significado patológico ainda não é completamente compreendido, mas por ser mais prevalente em pacientes idosos, hipertensos, diabéticos e tabagistas, sugere que alterações nas paredes dos vasos sanguíneos (hialinose) leve à hipoperfusão crônica e ao surgimento dessas lesões, podendo ser considerada, portanto, como uma isquemia subclínica. ${ }^{15}$ Recentemente, Kim e colaboradores ${ }^{15}$ estudaram se a SAOS poderia por si só justificar as alterações na substância branca cerebral. Para isso, submeteram mais de 500 pacientes à RNM de crânio e à polissonografia e após análise de dados e ajuste das covariáveis, demonstraram que a presença de AOS moderada a grave era fator de risco independente para a presença de lesões na substância branca cerebral.

Os potenciais mecanismos fisiopatológicos envolvidos no favorecimento da AVCi em pacientes com SAOS são as complexas alterações hemodinâmicas durante os episódios de apneia que levam a variações do fluxo cerebral e induzem lesão vascular por estresse de cisalhamento.15,16 Alterações bioquímicas também parecem estar envolvidas, sendo desencadeadas pela hipóxia intermitente crônica associada aos frequentes eventos respiratórios obstrutivos. Essa hipóxia intermitente promove estresse oxidativo e a ativação de vias pró-inflamatórias que em última análise promovem disfunção endotelial e um estado de hipercoagulabilidade que aceleram o processo de aterosclerose.,15

\section{Conclusão}

Ainda não estão totalmente esclarecidos os exatos mecanismos que levam a presença de SAOS a favorecer AVCi, mas surgem com estudos recentes cada vez mais evidências de que ela pode ser considerada um fator de risco independente para o desenvolvimento da doença cerebrovascular.

\section{Referências}

1. Zamarron C, Paz VC, Ribeiro A. Obstructive sleep apnea syndrome is a systemic disease. Current evidence. Eur $\mathrm{J}$ Intern Med. 2008;19(6):390-8. http://dx.doi.org/10.1016/j. ejim.2007.12.006

2. Franklin KA, Lindberg E. Obstructive sleep apnea is a common disorder in the population - a review on the epidemiology of sleep apnea. J Thorac Dis. 2015;7(8):1311-22.

3. Tufik S, Santos-Silva R, Taddei JA, et al. Obstructive sleep apnea syndrome in the Sao Paulo Epidemiologic Sleep Study. Sleep Med. 2010;11(5):441-46. http://dx.doi.org/10.1016/j. sleep.2009.10.005

4. Young T, Shahar E, Nieto FJ, et al. Sleep Heart Health Study Research Group. Predictors of sleep-disordered breathing in community-dwelling adults: the Sleep Heart Health Study. Arch Intern Med. 2002;162:893-900. http://dx.doi.org/10.1001/ archinte.162.8.893 
Christianne M.C.S. Bahia e cols • Síndrome da apneia obstrutiva do sono como risco independente de doenças cerebrovasculares

5. Darien IL. International classification of sleep disorders. 3rd ed. American Academy of Sleep Medicine; 2014.

6. Epstein LJ, Kristo D, Strollo PJ Jr, et al. Adult Obstructive Sleep Apnea Task Force of the American Academy of Sleep Medicine. Clinical guideline for the evaluation, management and long-term care of obstructive sleep apnea in adults. J Clin Sleep Med. 2009;5(3):263-276. http://dx.doi.org/10.1590/ S1980-57642015DN91000003

7. Ovbiagele B, Nguyen-Huynh MN. Stroke Epidemiology: Advancing our understanding of disease mechanism and therapy. Neurotherapeutics. 2011; 8(3):319-29. http://dx.doi. org/10.1007/s13311-011-0053-1

8. Peppard PE, Young T, Palta M, et al. Prospective study of the association between sleep-disordered breathing and hypertension. N Engl J Med. 2000;342: 1378-84. http://dx.doi. org/10.1056/NEJM200005113421901

9. Mehra R, Benjamin EJ, Shahar E, et al. Association of nocturnal arrythmias with sleep-disordered breathing. The Sleep Heart Health Study. Am J Respir Crit Care Med. 2006;173:910-16.

10. Monahan K, Storfer-Isser A, Mehra R, et al. Triggering of nocturnal arrhythmias by sleep-disordered breathing events. J Am Col Cardiol. 2009;54(19):1797-804. http://dx.doi.org/10.1016/j. jacc.2009.06.038

11. Béjot Y, Daubail B, Giroud M. Epidemiology of stroke and transient ischemic attacks: Current knowledge and perspectives. Rev Neurol (Paris). 2015 Dec 21. doi: 10.1016/j.neurol.2015.07.013.

12. Kernan WN, Ovbiagele B, Black Hr, et al. Executive Summary: Guidelines for prevention of stroke in patients with stroke ans transient ischemic attack. A guideline for healthcare professionals from the American Heart Association/American Stroke Association. Stroke. 2014:1-15.

13. Redline S, Yenokyan G, Gottlieb DJ, et al. Obstructive sleep apnea-hypopnea and incident stroke. The Sleep Heart health Study. Am J Respir Crit Care Med. 2010;182(2):269-77. http:// dx.doi.org/10.1164/rccm.200911-1746OC

14. Koo BB, Won C, Selim BJ, et al. The Determining Risk of Vascular Events by Apnea Monitoring (DREAM) study: design, rationale, and methods. Sleep Breath. Dec 2015;1-8. doi: 10.1007/s11325-015-1254-3. http://dx.doi.org/10.1007/ s11325-015-1254-3

15. Kim H, Yun $\mathrm{CH}$, Thomas RJ, et al. Obstructive sleep apnea as a risk factor for cerebral white matter change in a middle-aged and older general population. Sleep. 2013;36(5):709-715B.

16. Pizza F, Biallas M, Wolf M, et al. Nocturnal cerebral homodynamics in snorers and in patients with obstructive sleep apnea: a near-infrared spectroscopy study. Sleep. 2010;33(2):205-10. 